\title{
The Impact of a Teaching Activity on Cultural Competence Knowledge
}

Victoria Haynes

Department of Nursing, MidAmerica Nazarene University, Olathe, KS 66062-1899, United States

\begin{abstract}
Background: To determine if a teaching intervention can increase cultural competence knowledge in senior nursing students preparing to take the NCLEX-RN exam.

Methods: A 90-minute lunch and learn teaching intervention was conducted related to providing culturally competent care. A 10-question quiz was administered to a group of 31 senior nursing students before and after a teaching activity.

Results: Mean values for knowledge on cultural competency were significantly higher for the pretest compared to the posttest $(\mathrm{p}<0.01)$. There was a decrease in the NCLEX-RN pass-rate.

Conclusion: Though there was a decrease in the NCLEX-RN pass-rate, the teaching activity helped increase cultural competence knowledge in the nursing students on providing care to diverse patients. This implies that similar interventions may be used to increase knowledge related to cultural competence.
\end{abstract}

\section{Introduction}

Diversity is increasing at significant rates in the United States and is expected to continue to rise [1]. Immigration numbers, particularly from South American countries, have risen 25\% from 2007-2015 and the number of Black immigrants in the United States is rapidly increasing [2]. Racial and ethnic disparities are also more prevalent in diverse populations with notable findings in access and quality [3]. According to the 2014 Agency for Healthcare Research and Quality report, Black and Hispanic populations had worse access to care than Whites for over one half of the access measures and about one-third of the quality measures [3]. This increase in diversity and disparities that exists makes achieving cultural competency essential to ensure that all persons receive quality health care.

Nurses play a vital role in healthcare delivery. Nursing competence to practice is measured by the NCLEX-RN exam, an exam that measures the knowledge and skills of nurses and their readiness to care for patients and obtain licensure to practice [4]. The exam contains an area that assesses psychosocial social integrity that includes things like providing culturally competent care. A research project was conducted to determine if an educational intervention on providing culturally competent care could help increase cultural competence knowledge in traditional Bachelor of Science in Nursing (BSN) students.

\section{Background}

\section{Cultural competency education}

The need for cultural competency education in nursing programs is well-established. Mareno and Hart conducted a study to determine cultural competence among undergraduate and graduate nurses. They surveyed over 300 nurses using a prospective, cross-sectional, descriptive design. The found that, on average, undergraduate nurses scored significantly lower than graduate nurses related to cultural competence knowledge [5]. Both graduate and undergraduate nurses reported limited cultural diversity workplace training and continuing education, though undergraduates reported this at a higher rate. The findings support the need cultural competency education and areas of need for undergraduate and graduate nursing education. A descriptive, cross-sectional design was also used in a multicountry study conducted on over 2163 nursing students [6]. There were variations in cultural competency knowledge seen in different countries. Knowledge deficits were noted in the students' ability to discuss differences between health beliefs/behaviors and nursing knowledge seen with clients they serve [6]. Alpers and Hanssen conducted research using varied methods to determine cultural competence in practicing nurses. They found that there was an education gap related to cultural competence and concluded that adequate formal education is needed [7]. These findings support the need to adequately prepare nursing students to provide culturally competent care in nursing education programs.

\section{Circular approaches to cultural competency education}

A study was conducted by Kardong-Edgren \& CampinhaBacote on cultural competency of graduating BSN nursing students to determine the best curricular approach to teaching cultural competency. They evaluated the effectiveness of curricula in four different nursing programs related to developing culturally competent new graduates [8]. They found that no one approach is more effective in teaching cultural competency. Cultural competency courses and cultural competency integrated into the curricular both proved to be effective methods. A study by Long had similar findings. In this study the most common approaches to teaching cultural competency in nursing students were reviewed. These included lectures, group discussions, simulation, guest lecturers, mentoring/consultation, educational partnerships, and lived immersion/study abroad. The researcher did not find one approach that was more effective but did find that diverse clinical experiences, standardized patients, and immersion experiences, had a significant effect on the student's cultural awareness [9]. Kohlbry utilized a triangulated methodology to determine the impact of an international service learning project on cultural competency knowledge in nursing students. Immersion experiences were found to have a valuable impact on increasing cultural competency knowledge [10]. Studies like these supports the need for cultural competency educational interventions.

*Corresponding Author: Dr. Victoria Haynes, Department of Nursing, MidAmerica Nazarene University, Olathe, KS 66062-1899, United States, Tel: 913971-3833; E-mail: vmhaynes@mnu.edu

Citation: Haynes V (2018) The Impact of a Teaching Activity on Cultural Competence Knowledge. Int J Nurs Clin Pract 5: 302. doi: https://doi. org/10.15344/2394-4978/2018/302

Copyright: (C) 2018 Haynes. This is an open-access article distributed under the terms of the Creative Commons Attribution License, which permits unrestricted use, distribution, and reproduction in any medium, provided the original author and source are credited. 


\section{Reducing health disparities}

Preparing nurses and other healthcare providers to provide culturally competent care is essential to reducing racial and ethnic disparities. Like (2011) conducted an extensive literature review regarding disparities and cultural competency interventions. $\mathrm{He}$ found that continuing education for clinicians can play an important role in improving quality and reducing disparities [11]. Further examination of education programs is needed but the literature supports the need for cultural competency training, education, and interventions to help reduce disparities. Truong et al. (2014) conducted a systematic literature review of interventions to improve cultural competency, thus helping reduce disparities. The studies were categorized by study outcomes. The following three main categories were identified: patient-related outcomes, provider-related outcomes, and health service access/utilization outcomes. Though weak evidence for improvements in patient-related outcomes were found, there was a considerable amount of evidence that found improvements in the other outcomes [12]. These research studies help support that cultural competency interventions can be effective in reducing health disparities.

\section{NCLEX-RN and competency}

The effect of cultural competence education and NCLEX-RN scores is not well-established. Koestler (2015) evaluated comprehensive changes made to a baccalaureate nursing program to improve NCLEX-RN first-time pass rates. Technology improvements were made along with policy and outcomes assessment, student support, and curricular changes that proved to be effective in improving exam scores. The outcome of the curricular changes has led to significant improvements in pass rates from $72 \%$ in 2007 to $96.4 \%$ in 2011 [13]. Though the increase could have resulted from several factors, this intervention is promising that adding additional education to nursing curriculum can lead to an increase in NCLEX-RN exam scores. Davenport (2007) developed a similar comprehensive approach to prepare graduates for the NCLEX-RN. Curricular interventions were implemented each semester in an associate degree programs that were found to have positive effects on test-taking, content knowledge, critical thinking and confidence [14]. Though these studies are not specific to cultural competence interventions, they are promising that educational interventions can improve NCLEX-RN scores.

\section{Materials \& Method}

\section{Participants}

The participants in this study consisted of 31 senior traditional BSN nursing students enrolled in a nursing Senior Seminar: Transition to Nursing Practice course at midsize university located in a suburban town in Kansas. Twenty-eight of the students were female and three were male. Twenty-five of the students were White and six were nonWhite. A convenience sample of senior nursing students preparing to take the NCLEX-RN exam was selected to participate. Students who filled out a consent form and were in attendance in class that day were participants in the study. Students were informed that participation was voluntary and whether he or she choose to participate or not would have no effect on his or her grade or evaluation. The data for this project has been kept confidential. All of the student's information was de-identified and coded, and instructors cannot access identifiable data. Data has been stored on a locked, secure, flash drive accessible only by the researcher.

\section{Procedure}

A 10-question quiz was designed to assess their knowledge on cultural competence related to patient care before and after the teaching activity. The quiz questions were adapted from various cultural competency resources to help prepare nursing students to take the NCLEX-RN exam. Content validity for the quiz was provided by faculty members with experience with cultural competence. The pretest and posttest were administered in the Senior Seminar: Transition to Nursing Practice course before and after the 90-minute teaching intervention on providing culturally competent care. A pretest was given to the students to test their current knowledge on providing culturally competent care. The test was paper and pencil and consisted of 10 questions related to caring for diverse nursing clients. After the pretest, the researcher conducted a 90-minute educational intervention that covered content areas that are seen on the NCLEX-RN exam related to cultural competency. Visual aids such as a projector, and power point presentation were used to conduct the intervention. After the intervention, a posttest was given to assess the new knowledge that the students learned from the intervention. The pre and posttest contained identical questions and the test scores will be compared for each participant. After the intervention, the participants NCLEX-RN pass rates were compared with the preceding years TBSN at the university's pass rates. The limitation of this data is that the NCLEX-RN only reports pass rates in terms of pass/fail only so specific information related to psychosocial integrity was not available.

\section{Theoretical framework}

Philip Lister's taxonomy for developing cultural competence model (1999) was used to guide this project. In Lister's model, there are five elements that include cultural awareness, cultural knowledge, cultural understanding, cultural sensitivity, and cultural competence. Cultural awareness is the student's ability to describe their personal values and beliefs that are shaped culture and how different cultures and ethnicities can validate these [15]. The nursing students in this project were given a self-reflection activity to help determine their level of cultural awareness. They were also given definitions related to culture and performed an ice breaker activity to help then become familiar with the various cultures within their group. Cultural knowledge is when the student becomes familiar with the similarities and differences between various cultural groups [15]. In the teaching intervention the pre and posttest were used to assess the nursing students' level of cultural knowledge before and after the intervention and to determine if the intervention could increase cultural knowledge. Cultural understanding involves the student recognizing the issues faced by different cultures when they conflict with the dominant culture [15]. The teaching intervention highlighted some of the issues faced by different cultures and ways to assess these patients to provide quality nursing care. The students were given opportunities to share their nursing experiences that involved caring for patients from other cultures. Cultural sensitivity is when the student is able to show regard for the unique cultures of individuals that they care for and are aware of how their own culture may influence their professional practice [15]. Cultural competence is when the student is able to provide culturally competent care with respect for the values, beliefs, and practices of the patients they serve in relation to the dominant culture [15]. This teaching intervention was used to determine the level of cultural knowledge of the students and to help determine if an educational intervention on providing culturally competent care can help increase cultural competence knowledge. Data from the NCLEX$\mathrm{RN}$ results can also help determine knowledge increases. 


\section{Results}

A passing score on the pre-and post were set at eight out of ten or $80 \%$. Out of the 31 students, five scored an eight out of ten, three scored a nine out of ten and one scored ten out of ten on the pretest, making nine out of thirty-one or $29 \%$ passing the pretest. After the educational intervention, out of the 31 students 12 scored eight out of ten, five scored nine out of 10 and two scored 10 out of 10 . This means that 19 out of 31 or $61 \%$ passed the post test. The pass rate increase was $32 \%$. Paired $t$ tests were used to compare pre-and posttest scores. A statistically significant difference was found between the scores with a two-tailed $p$ value of 0.0017 . This shows that the educational intervention can help increase cultural competence knowledge. NCLEX-RN pass rate data was compared for this TBSN class with the previous graduating TBSN class results, first-time test takers. The data showed a decrease in the overall pass rates from $88.66 \%$ to $87.83 \%$. The limitation of this data is that it is unknown what other factors may have caused a decrease and if they were lower scores in other areas of the exam. The data is limited to pass or fail and does not give specific information regarding the scores and pass rates.

\section{Discussion}

The results of the study help support the need for education on cultural competency in nursing education. Though we were no changes in the overall NCLEX-RN pass rate, there was a significant increase in knowledge related to cultural competence. Additional research is needed in different nursing programs like graduate and practical nurse programs with larger sample sizes. There also needs to be more specific information from the NCLEX-RN to determine if this intervention is beneficial in increasing knowledge in the psychosocial areas. The pretest/posttest used was piloted on this group and needs to be used on additional groups to assess reliability and validity. The use of a tested tool with proven validity and reliability could also be helpful in future studies.

\section{Acknowledgements}

I would like to thank Susannah Hart for encouraging me to conduct this research project and for coordinating the TBSN students. I would also like to give a special thanks to my husband, my daughter, and my family for being my motivation to pursue my passion and for always supporting me.

\section{Competing Interests}

The author declare no competing interests.

\section{References}

1. United States Census Bureau (2010) 2010 census data.

2. Cilluffo A, Cohn D (2018) Seven demographic trends shaping the U.S. and the world in 2018. Pew Research Center.

3. Agency for Healthcare Research and Quality (2014) National Health Care Quality and Disparities Report.

4. National Council of State Boards of Nursing (2018) Creating the NCLEX \& Passing Standard.

5. Mareno N, Hart P (2014) Cultural competency among nurse with undergraduate degrees: Implications for nursing education. Nurs Educ Perspect 35: 83-88.
6. Cruz JP, Auinaldo AN, Estacio JC, Alotaibi A, Arguvanli S, et al. (2017) A multicountry perspective on cultural competence among baccalaureate nursing students. J Nurs Scholarsh 50: 92-101.

7. Alpers LM, Hanssen I (2014) Caring for ethnic minority patients: A mixed method study of nurses' self-assessment of cultural competency. Nurse Educ Today 34: 999-1004.

8. Kardong-Edgren S, Campinha-Bacote J (2008) Cultural competency of graduating US Bachelor of Science nursing students. Contemp Nurse 28: 37-44.

9. Long $T$ (2012) Overview if teaching strategies for cultural competence in nursing students. J Cult Divers 19: 102-108.

10. Kohlbry PW (2016) The impact of international service-learning on nursing students' cultural competency. J Nurs Scholarsh 48: 303-311.

11. Like R (2011) Educating clinicians about cultural competence and disparities in health and health care. J Contin Educ Health Prof 21: 196-206.

12. Truong $M$, Paradies $Y$, Priest $N$ (2014) Interventions to improve cultural competency in healthcare: A systematic review of reviews. BMC Health Services Research: 14: 99

13. Koestler D (2015) Improving NCLEX-RN first-time pass rates with a balanced curriculum. Nurs Educ Perspect 36: 55-57.

14. Davenport N (2007) A comprehensive approach to NCLEX-RN success. Nurs Educ Perspect 28: 30-33.

15. Lister P (1999) A taxonomy for developing cultural competence. Nurse Educ Today 19: 331-318. 